\title{
RETOS ACTUALES DE LAS CIENCIAS SOCIALES EN AMÉRICA LATINA
}

\section{CURRENT CHALLENGES FROM SOCIAL SCIENCES IN LATIN AMERICA ATUAIS DESAFIOS DAS CIÊNCIAS SOCIAIS NA AMÉRICA LATINA}

\section{Páginas Eduardo Restrepo}

98-109 eduardoa.restrepo@gmail.com

Antropólogo. Profesor asociado del Departamento de Estudios Culturales, Universidad Javeriana, Bogotá. 


\section{Resumen}

Las transformaciones de las ciencias sociales en los países de América Latina en los últimos tres o cuatro lustros han implicado dimensiones tecno-

lógicas y generacionales y se han sucedido en el marco de mayores incidencias de unas políticas de ciencia y tecnología abiertamente productivistas y del posicionamiento del modelo gerencial de la universidad-empresa. En este artículo se examinan estas transformaciones para sugerir una serie de retos a los que nos enfrentamos si el propósito es el de unas ciencias sociales críticamente contextuales y relevantes políticamente.

Palabras clave

Políticas de ciencia y tecnología, academización, cambios generacionales, politización, universidad-empresa 


\section{Abstract}

The transformation of social sciences in Latin American countries in the last three or four lustrums has involved technological and generational dimensions as it has happened in the context of most incidences of science and technology policies openly productive, and the positioning of the management model from the university-company. In this article these transformations are examined to suggest a number of challenges that we face if the purpose from the social sciences is critically contextualized and politically relevant.

\section{Key words}

Science and technology policies, academic action, generational changes, politicization, university-company

\section{Resumo}

As transformações das ciências sociais nos países da América Latina nas últimas três ou quatro lustros envolveram dimensões tecnológicas e geracionais assim como no contexto de maiores incidências de políticas de ciência e tecnologia abertamente produtivistas e do posicionamento do modelo gerencial da universidade-empresa. Neste artigo essas transformações são investigadas para sugerir uma série de desafios que enfrentamos se o objetivo é o de umas ciências sociais criticamente contextuais e relevantes politicamente.

\section{Palavras-chave}

Políticas de ciência e tecnologia, academização, mudanças geracionais, politização, universidade-empresa 
"[...] el hecho de que el conocimiento sea una construcción social también significa que es socialmen-

te posible tener un conocimiento más valido”. (Wallerstein, 1996, p. 101).

\section{Introducción}

Abrir las ciencias sociales (1996) es el sugerente título de un pequeño libro publicado a principios de los años noventa como resultado de un informe de la Comisión Gulbenkian para la reestructuración de las ciencias sociales, compuesta por destacados académicos como el antropólogo haitiano Michel Rolph Trouillot y el filósofo africano V.Y. Mudimbe, bajo la coordinación de Emmanuel Wallerstein. Este libro presenta la historia de la constitución de las ciencias sociales asociada a las transformaciones en el sistema-mundo moderno. Distinciones entre las esferas de lo político, el mercado y lo social, así como el doble contraste entre presente/pasado y nosotros/ellos definen las premisas desde las cuales emerge el modelo de división disciplinaria de la labor académica. Ahora bien, este modelo no fue el simple resultado de las dinámicas intrínsecas de configuraciones epistémicas, sino que claramente respondía a las particulares demandas de la expansión y reproducción colonial y neocolonial del sistema-mundo moderno hacia mediados del siglo XIX y gran parte del siglo XX.

Luego de presentar esta historia, los autores cierran su libro con un capítulo en el que se preguntan por el tipo de ciencia social que debería constituirse ahora, cuando las transformaciones económicas y políticas del sistema-mundo han puesto en cuestión gran parte de las premisas y de las condiciones de existencia del modelo de la división disciplinaria decimonónico. Su respuesta apunta hacia trascender las fronteras disciplinarias convencionales, así como romper con el estadocentrismo y positivismo dominantes sin caer en relativismos epistémicos y cinismos postmodernos. De ahí la urgencia de abrir las ciencias sociales.
En el cierre de la década del noventa, convocado por el Instituto de Estudios Sociales y Culturales Pensar, de la Universidad Javeriana, se realiza en Bogotá un simposio internacional "La reestructuración de las ciencias sociales en los países andinos”, que da origen a un libro titulado La reestructuración de las ciencias sociales en América Latina. Editado por Santiago Castro, la obvia relación del título con el libro coordinado por Wallerstein es corroborada en la Introducción en donde se hace referencia de manera detalla a los argumentos de ese libro. Además de confluir con Wallerstein en la urgencia de abrir las ciencias sociales cuestionando las premisas convencionales y las inercias institucionales que conservan las divisiones disciplinarias decimonónicas, Castro y Guardiola hacen énfasis, en la introducción del libro (Castro, 1999), en un enfoque que prioriza la geopolítica del conocimiento y en el cuestionamiento del eurocentrismo de las ciencias sociales dominantes. En la demanda de abrir las ciencias sociales se retoma el concepto de "reestructuración”, de ahí el título dado al seminario y al libro. En ambos casos agregando la marcación del lugar desde donde se habla: los países andinos para el seminario y América Latina en el libro.

Los cuestionamientos a las ciencias sociales, referidos a las limitaciones de los enfoques positivistas y al desenmascaramiento de su eurocentrismo, no aparecen con este libro a finales de los noventa. Por lo menos desde dos décadas atrás la pregunta por el colonialismo intelectual y una sociología propia ya se expresaba de manera clara en los trabajos de Orlando Fals Borda $(1979,1970)$. Antes que una voz aislada, Fals Borda perteneció a una generación de sociólogos, antropólogos e historiadores que no solo en Colombia, sino en diferentes países de América Latina, se preguntaron seriamente por el para qué, desde dónde y para quiénes de las ciencias sociales en los contextos y realidades propias. Las premisas de la neutralidad valorativa y de la objetividad, fueron ampliamente denunciadas como coartadas de un distanciamiento cínico agenciado por ciertos modelos metropolitanos de ciencias sociales. 
Obviamente, el lenguaje teórico, los alcances de la crítica, el momento histórico y lo que está en juego ha cambiado sustancialmente desde la década de los sesenta y los ochenta al momento en que se publica un libro como el editado por Castro-Gómez en el umbral del cambio de siglo. Lo único que quiero señalar con mencionar estos antecedentes es que es importante no perder de vista que las preocupaciones y cuestionamientos a las ciencias sociales en los países de América Latina y el Caribe tienen densas genealogías, las cuales en gran parte todavía estamos en mora de trazar como memorias de las luchas que han configurado cómo hemos llegado a ser lo que somos.

No es mi intención en este artículo hacer una contribución a esta genealogía. Me he referido al libro coordinado por Wallerstein y al editado por Castro-Gómez para argumentar que, aunque sigue siendo indispensable abrir las ciencias sociales y hacerlo teniendo en consideración la dimensión geopolítica del conocimiento y la superación de la arrogancia-ceguera eurocéntrica, hoy asistimos a una serie de transformaciones en el campo de la ciencias sociales en los distintos países de América Latina que no podemos dejar de tomar en consideración. Pienso que, en algunos aspectos nodales, estas transformaciones introducen inflexiones sustantivas a la labor de abrir-reestructurar-repotenciar las ciencias sociales en nuestros países.

En el siguiente aparte identificaré cuatro de estas transformaciones, las cuales se encuentran profundamente imbricadas: transformaciones tecnológicas, generacionales, en los efectos de las políticas de ciencia y tecnología, y en el posicionamiento del modelo gerencial de universidad-empresa. Con esta perspectiva panorámica, cierro este artículo con la referencia a una serie de retos que se imponen hoy en el campo de las ciencias sociales en América Latina.

\section{Transformaciones}

En los últimos tres lustros son muchas las transformaciones que se han suscitado en el campo de las ciencias sociales en un país como Colombia. Referirme a estas transformaciones es hablar de cambios que he atestiguado en mi trayectoria como antropólogo y practicante de estudios culturales. Algunos cambios se han dado sutilmente, sin mayores sobresaltos, otros se han introducido de manera más abrupta y evidente. No obstante, estos ritmos diferenciales, lo que implicaba hacer parte de una disciplina como la antropología o participar de un campo transdisciplinario como los estudios culturales ha cambiado sustancialmente en los últimos años al menos en cuatro aspectos.

El primero se refiere a las transformaciones tecnológicas que han modificado sustancialmente una parte importante de las prácticas que constituían la labor cotidiana de la producción, circulación o apropiación de las ciencias sociales en nuestros contextos. Las nuevas tecnologías computacionales han modificado las maneras en las cuales se adelanta gran parte de la producción de conocimiento en ciencias sociales, desde el acceso a las bases de datos hasta la posibilidad de compartir documentos a los colegas en fracciones de segundo, quienes, a su vez, los pueden leer y comentar inmediatamente. Los grupos de investigación pueden intercambiar datos y elaboraciones en plataformas diseñadas para esto o simplemente a través de redes sociales o listados de correo electrónico. En muchos aspectos se ha potenciado sustantivamente la posibilidad de identificar, almacenar y analizar un volumen de información como nunca antes había sido siquiera soñado. Ya sea como instrumentos o como metodologías para la investigación o para la visibilización de los resultados o debates, con las nuevas tecnologías computacionales hoy enfrentamos nuevos constreñimientos y posibilidades. 
Estas transformaciones tecnológicas no solo suponen un cambio en muchas de las prácticas de la investigación, apropiación y circulación de las ciencias sociales, sino que también devienen en importantes temáticas de estudio. Nuevas temáticas que abren todo un nuevo continente, el de la cibercutlura, el de las redes sociales, pero también viejas temáticas que pueden ser pensadas desde otros ángulos, como las identidades, las corporalidades, las espacialidades.

Abrir-reestructurar las ciencias sociales tiene que tomar seriamente en consideración la manera como nuestras prácticas más cotidianas se han visto atravesadas por la creciente dependencia de estas tecnologías. Nuevas sujeciones, otros amarres de las geopolíticas, pero también cambios sustanciales en qué significaría hoy el lugar de enunciación, la marcación de lugar, asociadas a las competencias-dependencias tecnológicas deben ser consideradas. No basta con abrir-reestructurar las ciencias sociales quedándose en un plano epistemológico; hay que considerar seriamente la dimensión de las prácticas que constituyen las ciencias sociales, y en esta dimensión las transformaciones tecnológicas no pueden dejarse de lado.

Una segunda transformación es la generacional. Esta transformación no solo se evidencia en el perfil del grueso de los estudiantes que hoy se involucran en estudiar ciencias sociales, sino también en los relevos generacionales que se están sucediendo en sus practicantes tanto en la academia como en los otros ámbitos de la práctica profesional. Hace dos décadas, el perfil predominante de los estudiantes de las carreras de ciencias sociales era muy diferente de quienes hoy se encuentran matriculados.

Aunque las ciencias sociales siguen, en muchos aspectos, siendo marginales en la economía de los prestigios que opera en el grueso del imaginario social, sí ha habido un cambio en cuanto a las expectativas de quienes se matriculan en estas disciplinas. Los jóvenes llegan y se gradúan con la esperanza de que con sus estudios obtendrán un trabajo con el estado, las empresas o el sector de las fundaciones y ONG. Muchos de estos jóvenes son financiados y viven con sus padres, y ven en sus estudios fundamentalmente una opción profesional de la cual puedan derivar su sustento económico. Las discusiones éticas o políticas sobre el quehacer y razón de ser de las ciencias sociales, que eran candentes hasta hace unos años, ahora son vistas con cierta extrañeza o con franco aburrimiento por muchos de los jóvenes estudiantes. Pensar su labor orientada hacia el mercado o hacia el estado no es un asunto que los conflictúe, sino más bien el horizonte de sus deseos. Esto contrasta con una o dos décadas atrás donde la elección de estudiar una disciplina en ciencias sociales estaba inspirada en criterios muy distintos, entre los cuales eran preponderantes los intereses políticos o humanísticos (por llamarlos de alguna forma).

Con respecto a los practicantes más visibles en el establecimiento académico y en otros escenarios del desempeño disciplinar, también se han suscitado transformaciones sustanciales en los últimos tres lustros. Uno de los más visibles es que la generación que experimentó unas ciencias sociales críticas en las décadas de los setenta y ochenta se ha ido jubilando o ha ido cediendo paso a colegas más jóvenes formados en la década del noventa o la primera del dos mil, que han obtenido sus titulaciones de postgrado a menudo en academias metropolitanas. Este relevo generacional también es de estilo y de ethos, dándosele más prioridad a unas disciplinas cada vez más academizadas en lo que se refiere al establecimiento universitario y, sorprendentemente, mucho más dóciles a las múltiples demandas del mercado y de la gubernamentalización de la existencia de la vida social.

En suma, preocupaciones sobre la geopolítica del conocimiento, las políticas de la teoría o el compromiso suenan distantes, poco seductores, cuando no absolutamente inconmensurables con los intereses y móviles que interpelan en términos generales a 
la generación de estudiantes y practicantes de las ciencias sociales. Así que si el propósito es abrir o reestructurar las ciencias sociales en América Latina uno no puede darse el lujo de desconocer que hoy el grueso de la generación de estudiantes y practicantes son, a menudo, interpelados por unas expectativas y horizontes de profesionalización que en el mejor de los casos se muestran indiferentes, cuando no abiertamente reacios, a considerar los constreñimientos e implicaciones de lo que aparece ante sus ojos y reproducen en sus prácticas como ideales de las ciencias sociales.

La tercera transformación es la referida a los efectos de las políticas de ciencia y tecnología agenciadas por las entidades estatales. Hubo una vez un tiempo donde las prácticas de los profesores universitarios y los investigadores en las ciencias sociales en general no estaban atravesadas por las normatividades y por los efectos de las políticas de ciencia y tecnología que impulsan las entidades estatales en nuestros países; y esos tiempos, así a muchos les parezcan remotos y extraños, existieron en países como Colombia hasta hace solo un par de décadas atrás. Quienes hemos habitado los escenarios académicos desde hace al menos veinte años, tenemos una perspectiva diferente sobre lo que hoy parece imponerse como un hecho naturalizado: hubo una época en la cual no imperaban los cvlacs y gruplacs, ${ }^{1} \sin$ la obsesión por revistas indexadas en ISI o Scopus, sin la regulación, por la burocracia académica, de los procesos de investigación, definidos desde proyectos y medidos en resultados como publicaciones.

En Colombia, por ejemplo, estas transformaciones se han ido posicionando lentamente. La clasificación de los grupos de investigación y el registro de la producción de los académicos en sus cvlacs son medidas que han sido incorporadas por cada vez

1 Estos son los nombres que la entidad estatal en Colombia (Colciencias) le da a la estandarización de las hojas de vida de los académicos y de los grupos de investigación, respectivamente. más universidades e investigadores. Las diferentes burocracias académicas de universidades y otras entidades donde se adelanta investigación han presionado indirecta o directamente a los profesores e investigadores para que su labor responda a la lógica de medición establecida por Colciencias, que ha entronizado la cienciometría. Se ha desatado una especie de ansiedad de la clasificación por estar en las mejores posiciones, con números de grupos de investigación en $\mathrm{A}+\mathrm{o}$ porque sus revistas sean indexadas y reconocidas. Todo esto aún opera dentro de la lógica de recompensas simbólicas que, dependiendo de las universidades o entidades de investigación, se pueden traducir o no en bonificaciones o escalonamiento. En mucho se mantiene el mecanismo de la "carita feliz", la recompensa simbólica, dado que Colciencias normatiza y mide pero no financia realmente la investigación ni a los investigadores en el país. ${ }^{2}$

Para legitimar las tecnologías normalizantes de las prácticas de los académicos e investigadores, la burocracia en Colciencias ha apelado a un discurso de la eficiencia, productividad, calidad, competitividad e internacionalización. Los imaginarios y supuestos de este discurso son grotescamente obvios: “internacionalización”, por ejemplo, es plegarse irreflexivamente a una particular geopolítica del conocimiento donde unos centros, lenguajes, modalidades de argumentación, formas de visibilización y audibilidad son dominantes. Como lo hemos trabajado para el caso concreto de la antropología, esto supone reproducir ciertas políticas de la ignorancia, perpetuar unos silenciamientos y asimetrías (Ribeiro y Escobar, 2008; Restrepo, 2012).

2 Esto contrasta con los modelos del Conicet en Argentina o del Conacyt en México, que cuentan con reales programas de financiación (no simplemente con convocatorias a becas o a la financiación de ciertos proyectos). Tal vez el ejemplo más extremo es el de Chile, donde el Conicyt ha introducido un mecanismo de pago en dinero a las universidades por artículo publicado en revistas indexadas en ISI o Scopus, lo que ha hecho que las universidades establezcan modalidades de presión-incentivación a sus profesores e investigadores para que orienten sus labores hacia la publicación de estos artículos. 
Poco se discuten los criterios desde los cuáles se mide, qué se mide, quién mide y para qué se mide. Son marginales las iniciativas de investigación y debate sobre sus efectos en la transformación de las prácticas académicas. Entre los efectos más visibles, sin embargo, está la fetichización del creciente productivismo (número de artículos publicados o patentes solicitadas) asociada a una marcada despolitización de las labores investigativas que se subsumen cada vez más abiertamente en responder a demandas empresariales. Así las cosas, el propósito de abrir o reestructurar las ciencias sociales en América Latina no puede soslayar los efectos de las políticas de ciencia y tecnología que se han ido posicionando en nuestros países en los mapas de interés, en las prácticas y en las subjetividades de quienes hacen ciencias sociales.

Estrechamente asociada a estas políticas estatales encontramos la cuarta transformación: la consolidación del modelo gerencial de la universidad-empresa. Si bien las universidades privadas en nuestros países se han concebido desde la lógica empresarial de la ganancia, lo que ha cambiado en las últimas dos décadas es la generalización del modelo gerencial en universidades públicas y privadas, donde se imponen cada vez más las preocupaciones por la rentabilidad de los programas ofrecidos y la transformación de los estudiantes en clientes. Las burocracias universitarias han ido extendiendo su accionar con la creciente regulación de las prácticas académicas a través de la imposición de una filigrana de procedimientos y formatos a los que cada vez se hace más difícil escapar y que tienden a consumir más tiempo y dedicación.

Así, la investigación se ha convertido en una actividad fuertemente regulada y estructurada por demandas de la burocracia académica. Para solicitar recursos o descarga de tiempo, el investigador se ve compelido a operar dentro de una serie de requerimientos de ritmos y productos fijados por las burocracias universitarias. Las consultorías se imponen cada vez más como el escenario de la investi- gación, lo que la subsume a los intereses de quienes contratan.

Estas regularizaciones sobre las labores de los docentes e investigadores en las universidades evidencian una transformación sustancial en la concepción misma de la universidad. De una idea de universidad articulada por la función ético y política humanista de devenir en instancia para la reflexión crítica y autónoma de la sociedad, se ha ido naturalizando una noción de universidad orientada por una racionalidad instrumental tendiente a producir los tecnócratas y expertos que requiere el mercado y el estado. El modelo gerencial imperante es la punta del iceberg de los profundos cambios que se han suscitado en la universidad, incluso en aquellas que aún se siguen considerando como públicas. $^{3}$

El propósito de abrir o reestructurar las ciencias sociales no puede desconocer estas transformaciones que, desde la cotidianidad misma, troquelan los alcances y limitaciones reales de las ciencias sociales hoy en nuestros países. Así, por ejemplo, la creación de postgrados en estudios culturales en Colombia no es necesariamente un indicio de la apertura de las ciencias sociales o de su reestructuración, como lo sugieren en los libros citados de Wallerstein y Castro-Gómez, respectivamente; al contrario, la creación de estos programas responde en mucho a las expectativas de las burocracias universitarias y es perfectamente funcional a las transformaciones del sistema universitario en el país, respondiendo a las políticas de ciencia y tecnología ya señaladas.

3 El proceso de privatización de las universidades públicas no hay que entenderlo simplemente desde la perspectiva de dónde provienen los recursos para su funcionamiento y quiénes pueden acceder a sus programas; también hay que considerar el modelo mismo de universidad, del para qué, el cómo y el hacia dónde que se expresa en prácticas como el posicionamiento del modelo gerencial comentado. 


\section{Retos}

He argumentado que se puede identificar una serie de transformaciones en los tres o cuatro últimos lustros que han significado cambios importantes de los términos en los cuales se despliega el campo de las ciencias sociales en distintos países de América Latina. A partir de este panorama, nos encontramos ante una serie de retos si lo que buscamos es potenciar unas ciencias sociales críticamente contextuales y relevantes políticamente.

El primer reto consiste en reorientar nuestra labor a partir de la profundización de los diálogos sursur. El colonialismo intelectual que se ha afianzado en las últimas décadas al tomar como referentes privilegiados a los autores, conceptualizaciones y estilos académicos euro-estadounidenses, requiere ser revertido por un giro en los términos de la conversación, al igual que en las genealogías intelectuales e interlocutores que nos interpelan y constituyen (Santos, 2009).

El reto de profundizar los diálogos sur-sur no se entiende como una apelación chovinista ni como un auto-orientalismo que supone un privilegio epistémico a las márgenes y lugares históricamente subalternizados; este reto lo entiendo más en la línea del historiador subalternista Dipesh Chakrabarty (2008), para quien el pensamiento europeo es indispensable pero insuficiente para comprender adecuadamente a las sociedades modernas coloniales como la India pero también a la misma Europa. De ahí su contundente planteamiento de la urgencia de provincializar a Europa.

Profundizar los diálogos sur-sur requiere de esta provincialización de Europa, pero no se limita a eso. Al mismo tiempo, hay que tomar en serio interlocutores que conversan en otros lenguajes, que suponen diferentes estilos intelectuales. Dejarse contaminar por su indisciplina, por su indocilidad, por lo herético de ritmos que no se pliegan a los imperativos de la productividad.
Un segundo reto, en ciertos sentidos asociado al primero, se refiere al posicionamiento del trabajo colectivo y colaborativo, así como al socavamiento del fetichismo del copyright. Los discursos de la productividad y competitividad que marcan fuertemente a las ciencias sociales en los últimos lustros han reforzado la figura del autor-individuo y cierta idea de los derechos intelectuales que concibe al conocimiento como una mercancía. Apostarle al trabajo colectivo y colaborativo en ciencias sociales, pareciera ser hoy una práctica que puede cuestionar la lógica del productivismo, debido a que los ritmos, prioridades y logros que tiende a involucrar este tipo de trabajo, contrastan con las lógicas de la acumulación productivista. Igualmente, se hace cada vez más importante desnaturalizar la concepción de los resultados del conocimiento como propiedad privada - a la que solo se puede acceder si se tiene cómo pagar-, que se produce en un escenario de competencia donde se premia el egoísmo y la apropiación individualizada, sancionada mediante la figura del copyright. Hay que explorar los alcances y límites de otras modalidades de propiedad intelectual como el creative commons y el copyleft, sin ser ingenuos con respecto a las sorprendentes habilidades del mercado y los intereses empresariales de cooptar iniciativas que se presentan como alternativas.

Cierta manera de entender la interdisciplinariedad constituye un tercer reto que enfrentan las ciencias sociales en países como el nuestro. En gran parte apuntalada por la burocracia académica, que busca reducir costos de operación, y en asocio al socavamiento de los pregrados, una errática concepción de la interdisciplinariedad se ha impuesto en no pocas universidades. De unos programas de formación estructurados en la apropiación de las tradiciones y estilos disciplinarios, hoy los estudiantes se encuentran los cuatro o cinco primeros semestres (cuando no más) recibiendo básicamente cursos de diferentes disciplinas desde una narrativa celebratoria de la interdisciplinariedad. 
Esto abarata los costos de los programas, pues un profesor se encarga de muchos más estudiantes; no obstante, es realmente una muy mala estrategia de interdisciplinarización, porque cada profesor sigue hablando desde sus lugares disciplinarios mientras que los estudiantes no cuentan con insumos sólidos ni de la disciplina a la que se matricularon y de la cual serán futuros profesionales ni de las otras disciplinas que caen como un chaparrón del cielo. La interdisciplinariedad no es un punto de partida sino de llegada. Es un horizonte de problematización de certezas disciplinarias. Puede haber descentramientos y auténticos diálogos cuando se cuenta con puntos que descentrar y lugares desde los cuales dialogar. ${ }^{4}$ Sin esto, lo que hay es una interdisciplinaridad cosmética basada en una floja o nula formación disciplinaria.

Mi argumento no es la reificación de las disciplinas para apelar a un patriotismo disciplinario ni la negación de la relevancia de la interdisciplinariedad; es más, en nociones como las de transdisciplinariedad e indisciplinariedad se pueden articular pertinentes procesos que permitan potenciar unas ciencias sociales críticamente contextuales y relevantes políticamente. Simplemente quiero indicar que la manera cómo se entiende la interdisciplinariedad por las burocracias académicas no es acertada por los efectos socavantes en la formación de las nacientes generaciones, por lo que uno de los retos pasa por tomarse en serio las disciplinas en aras de ir más allá de ellas.

Un cuarto reto, y tal vez uno de los más complicados, es el de posicionar nuevamente la relevancia de politizar las ciencias sociales. Con el desplazamiento hacia el devenir expertos, desde la lógica del productivismo academicista o desde la satisfac- ción dócil de las demandas del mercado y del estado, no son pocos los académicos que derivan su comodidad y ciertos privilegios que, con el tiempo y la perdida de perspectiva, no son fáciles de abandonar. Inscribir las ciencias sociales en el horizonte de la utopía, de la preocupación de un para qué y para quiénes que se articule con fuerzas y movilizaciones políticas reales, puede ser una tarea poco seductora para un sector de los académicos que, por razones que no se pueden examinar aquí, se identifican con el actual orden de las ciencias sociales en nuestros países. También es una propuesta poco atractiva para estudiantes cuyo propósito existencial es conseguir un 'buen' trabajo, sin mayores preocupaciones éticas o políticas más allá de su bienestar económico y social inmediato. El peso de la inercia del establecimiento académico, como se ha ido sedimentando en los últimos lustros, conjugado con la abierta resistencia de sus guardianes, hace titánico revertir la tendencia de unas ciencias sociales engolosinadas con dispositivos de recompensa inmediatos del productivismo y de su instrumentalización en el mercado y en la afinación de las tecnologías de sometimiento. No obstante, este es precisamente uno de los retos que hoy enfrentamos: la transformación de la imaginación teórica e histórica que articule la labor de las ciencias sociales a producir, compartir y contrastar conocimientos críticamente contextuales y relevantes políticamente.
4 Con Stuart Hall (2010), se puede argumentar que la interdisciplinariedad es indispensable, pero demanda una práctica rigurosa. Como solía decir: la interdisciplinariedad supone el reto de hacer mejor sociología que los sociólogos porque se toma en serio esta formación disciplinaria para descentrarla desde preguntas y estilos de trabajo heréticos a sus tradiciones. 


\section{Referencias}

Castro-Gómez, S. (1999). La reestructuración de las ciencias sociales en América Latina. Bogotá:

Pontificia Universidad Javeriana.

Chakrabarty, D. (2008). Al margen de Europa: pensamiento poscolonial y diferencia histórica. Barcelona: Tusquets.

Fals-Borda, O. (1979). El problema de cómo investigar la realidad para transformarla: por la praxis. Bogotá: Ediciones Tercer Mundo. . (1970). Ciencia propia y colonialismo intelectual. México: Editorial Nuestro Tiempo.

Hall, S. (2010). Sin garantías: trayectorias y problemáticas en estudios culturales. Lima: Envión editores-Universidad Andina Simón Bolívar-Instituto de Estudios Peruanos.

Restrepo, E. (2012). "Hacia una antropología crítica de la antropología”. Antropología y estudios culturales: disputas y confluencias desde la periferia. pp. 21-120. Buenos Aires: Siglo XXI Editores.

Ribeiro, G. L. \& A. Escobar. (2008). Antropologías del mundo: Transformaciones disciplinarias dentro de sistemas de poder. Popayán: Ciesas-Envión Editores.

Santos, B. de S. (2009). Una epistemología del sur: la reinvención del conocimiento y la emancipación social. México: Siglo XXI, CLACSO.

Wallerstein, I. et al. (1996). Abrir las ciencias sociales: informe de la Comisión Gulbenkian para la reestructuración de las ciencias sociales. México: Siglo XXI. 